\title{
Solar Atmospheric Neutrino searches with the ANTARES neutrino telescope
}

\section{Lopez-Coto ${ }^{1, *}$, S. Navas ${ }^{1}$ and J.D. Zornoza ${ }^{2}$ on behalf of the ANTARES Collaboration}

(a complete list of authors can be found at the end of the proceedings)

${ }^{1}$ University of Granada

Dpto. de Física Teórica y del Cosmos \& C.A.F.P.E., Av. Hospicio 18071, Granada, Spain.

${ }^{2}$ IFIC - Instituto de Física Corpuscular

CSIC - Universitat de València,

c/ Catedrático José Beltrán, 2 E-46980 Paterna, Valencia, Spain.

E-mail: daniellc@ugr.es

The interaction of cosmic-rays with the solar atmosphere can yield neutrinos as final state particles, the so-called Solar Atmospheric Neutrinos (SA $v$ s). Most of these neutrinos are absorbed in the interior of the Sun. Neutrinos produced in the solar corona towards the Earth would escape the Sun and reach the Earth. The detection of the solar atmospheric neutrinos would be important to determine the constituents of the primary cosmic rays and the solar composition. In addition, these neutrinos would represent an irreducible source of background for indirect solar dark matter searches. The deep-sea neutrino telescope ANTARES, located in the Mediterranean Sea, is well suited to perform this search. In this work, the results after the analysis of 11 years of ANTARES data is presented. No evidence for a solar atmospheric neutrino signal over the expected background is found. Results in terms of sensitivity and upper limits for different signal models are reported. The obtained upper limit at $90 \% \mathrm{CL}$ in the solar atmospheric neutrino flux is $7 \times 10^{-11}\left[\mathrm{TeV} \mathrm{cm}^{-2} \mathrm{~s}^{-1}\right]$ at $\mathrm{E}_{v} \sim 1 \mathrm{TeV}$.

$37^{\text {th }}$ International Cosmic Ray Conference (ICRC 2021)

July 12th - 23rd, 2021

Online - Berlin, Germany

\footnotetext{
${ }^{*}$ Presenter
} 


\section{Introduction}

In their way to the Earth, some cosmic rays (CRs) are blocked by the Sun. The interaction of these blocked CRs with the solar atmosphere may yield neutrinos in all directions. Some of these neutrinos would be absorbed by the inner part of the Sun, but others, those produced in the corona, can escape the solar medium and reach the Earth. These Solar Atmospheric Neutrinos (SA $v \mathrm{~s})$ would be an unavoidable background for dark matter (DM) indirect searches.

Besides the important fact that would be the understanding of the characteristics of this potential background source, the detection of these SA $\nu$ s can shed light on: the primary CR composition, the solar density and the parameters of neutrino oscillations [1,2].

The mechanism to produce SA $v$ s is similar to that of the production of atmospheric terrestrial neutrinos but, being the solar atmosphere less dense than the terrestrial one, the unstable secondary particles produced by CR interactions are more likely to decay than to interact in the solar medium, making the expected SA $v$ flux to be a slightly above than the atmospheric terrestrial one. The overall SA $v$ s are produced in the outer part of the Sun since at a sufficently large depth, almost every secondary CR would have decayed. Finally, the resulting neutrino ${ }^{1}$ flux at production has an approximate flavour ratio of $\left\{v_{e}\right\}:\left\{v_{\mu}\right\}:\left\{v_{\tau}\right\}=1: 2: 0$. However, the final neutrino flux at Earth, after oscillations, has a flavour ratio of $1: 1: 1[1]$.

\section{The ANTARES Neutrino Telescope}

The ANTARES detector [3] is anchored to the seabed at a depth of $2475 \mathrm{~m}$ in the Mediterranean Sea at $\left(45^{\circ} 45^{\prime} \mathrm{N}, 6^{\circ} 10^{\prime} \mathrm{E}\right), 40 \mathrm{~km}$ offshore from Toulon. ANTARES deployed its first detection line in 2007 and was completed in 2008. Since then, it has been continuosly taking data.

The ANTARES full configuration consists on 12 detection lines $450 \mathrm{~m}$ long distributed on an octogonal layout with an horizontal spacing of about 60-75 m. Each line houses 25 storeys, vertically spaced by about $14 \mathrm{~m}$. The $12^{\text {th }}$ line has 20 storeys completed with acoustic detection devices. Each storey hosts three optical modules (OMs), being the first one located $100 \mathrm{~m}$ above the sea-bed.

The OM [4] main component is a 10-inch hemispherical photomultiplier tube (PMT) glued in a pressure resistant glass sphere with optical gel. Each PMT is facing $45^{\circ}$ downward, optimizing the detection of upward-going light from charged particles.

The photons detected in the PMTs induce a signal called hit [5]. The position, time and collected charge of the hits are used to reconstruct the direction and energy of each event. Different trigger algorithms [3] are responsible for signal and noise classification.

The trigger and data acquisition in an under sea neutrino telescope is affected by environmental changes, bioluminescence processes, sea current velocity changes and possibly malfunctioning detector elements. In order to correctly reproduce the detector response under these conditions, ANTARES simulates the atmospheric muons and neutrino interactions following a MC run-by-run strategy [6].

\footnotetext{
${ }^{1}$ Here and in the following, the word neutrino refers to both $v$ and $\bar{v}$ unless otherwise specified
} 


\section{Analysis}

\subsection{Monte Carlo and Data sample}

Neutrino induced events can be classified into two main groups: track like and shower like events. Track like events are produced by charged current (CC) $v_{\mu}$ and $v_{\tau}$ interactions. $v_{\mu}$ CC interactions yield muons as final state particles. $v_{\tau} \mathrm{CC}$ interactions can produce final state muons through muonic decay of the final state particle $\tau$. All neutral current (NC) reactions, as well as charged current reactions of $v_{e}$ and most $v_{\tau}$, produce shower like events. These showers are typically several meters long and therefore small compared to inter-OM distances.

In this work we have tested two different CR models as signal component: the Hillas-Gaisser 3-generation model ( $\mathrm{H3a}$ ) [7] and the Gaisser-Stanev-Tilav 4-generation model (GST4) [8]. Also, two different solar density profiles have been used: Ser+Stein [9] and the Grevesse \& Sauval solar density profile, refered as Ser+GS98 [10]. These models are included within the solar_crnu WIMPSim 5.0 package [11, 12]. Neutrino oscillations from best-fit values [13] and normal mass ordering are assumed. Three different source shapes have been considered: the Sun as a point source, as a filled disk and with ring shape. As a base-line case of study the $H 3 a \mathrm{CR}$ model with the Ser+Stein solar density profile has been chosen. Also, the Sun has been considered as a point source.

The two main background sources considered for this analysis are atmospheric muons and atmospheric neutrinos produced in the interactions of cosmic rays in the upper atmosphere.

In this analysis, the search for SA $v$ s is done through the track channel only $\left(v_{\mu}\right.$ CC) taking advantage of the excellent ANTARES angular resolution $\left(0.4^{\circ}\right.$ at $\left.\mathrm{E}_{v}=10 \mathrm{TeV}\right)$. Also, a region of interest (RoI) of $30^{\circ}$ around the Sun is chosen. In order to optimize the search for SA $v$ signatures and reject the background, a selection of quality cuts $\left(\theta_{\text {zenith }}, \Lambda, \beta\right)$ [14] is applied. Selecting only upward-going events in the detector $\theta_{\text {zenith }}>90^{\circ}$, the background is reduced considerably because the atmospheric muons are stopped by the Earth. Cuts in the reconstruction fit parameter $\Lambda>-5.2$ and in the error estimate in the reconstructed angle $\beta<1^{\circ}$ are also established to select the best possible reconstructed events in the sample.

\subsection{Likelihood}

In order to search for an excess of signal of solar atmospheric neutrinos, an unbinned likelihood method based on the Neyman [15] approach is used. The likelihood used for the analysis is

$$
\mathcal{L}\left(n_{\mathrm{sig}}\right)=e^{-\left(n_{\mathrm{sig}}+n_{\mathrm{bkg}}\right)} \prod_{i}^{N}\left[n_{\mathrm{sig}} \cdot \mathcal{S}\left(\Psi_{\odot, i}, \beta_{i}, E_{i}\right)+n_{\mathrm{bkg}} \cdot \mathcal{B}\left(\Psi_{\odot, i}, \beta_{i}, E_{i}\right)\right],
$$

where:

- $\mathcal{S}$ and $\mathcal{B}$ are the signal and background PDFs, respectively.

- $n_{\text {sig }}$ is a free parameter to fit in the likelihood, and represents the number of signal events in the sample.

- $n_{\mathrm{bkg}}$ is the expected number of background events in the sample. 
- $N$ is the total number of reconstructed events within the RoI in the data taking period. It can be expressed as $N=n_{\text {sig }}+n_{\text {bkg }}$

- $\Psi_{\odot}$ is the angular distance to the source.

- $\beta$ is the error estimate in the reconstructed angle.

- $E$ is the energy proxy.

The information characterising the signal and background events is contained in their corresponding PDFs, $\mathcal{S}$ and $\mathcal{B}$ respectively. In Fig. 1 the PDFs used in the likelihood maximisation process are shown. In these PDFs the angular distance between the direction of the reconstructed track and the position of the Sun ( $x$-axis) is plotted as a function of the reconstructed energy of the event ( $y$-axis). The logarithm of the probability for an event to have a certain energy and angular distance to the source is shown in the colorbar. The PDF on the left shows that the signal events are highly probable to be accumulated above $10^{2.5} \mathrm{GeV}$ within a few degrees around the source, whilst background events are more uniformly distributed in angular distance but are more likely to have an energy between $10^{2}$ and $10^{3.5} \mathrm{GeV}$.

The signal PDF is built from Monte Carlo simulations [6] and the events are weighted by the Solar Atmospheric Neutrino energy spectra [1], which is computed with the package solar_crnu included in WIMPSIM 5.0 [11, 12]. The background PDF is built from scrambled data. The ingredients of each PDF are: the angular distance to the source, $\Psi_{\odot}$; the error estimate in the reconstructed angle, $\beta$; and the energy proxy, $E$. The likelihood maximisation process runs over the number of reconstructed events $\left(N=n_{\mathrm{sig}}+n_{\mathrm{bkg}}\right)$ within a region of interest (RoI) of $30^{\circ}$ around the source. The output best-fit parameter of the likelihood is $\hat{n}_{\text {sig }}$.

The significance of the signal event is established by the test statistic TS (Eq. 2).
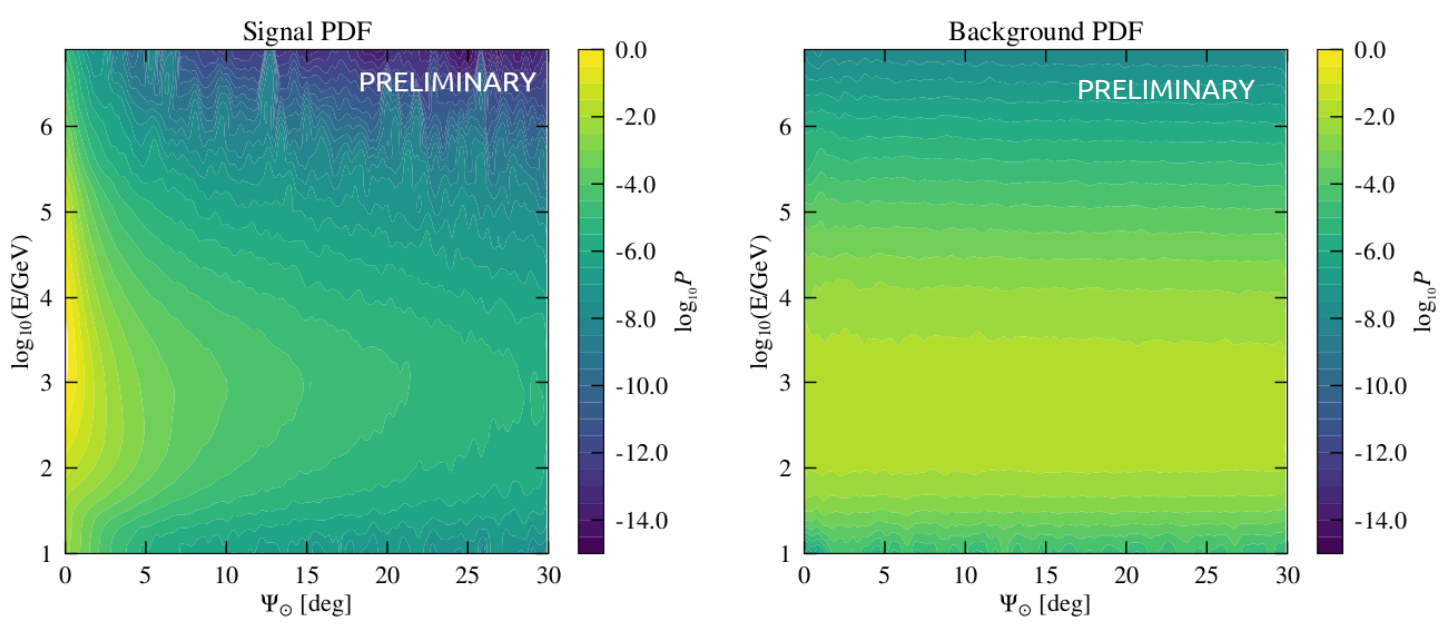

Figure 1: Signal (left) and background (right) PDFs used as inputs in the likelihood function. The angular distance between the direction of the reconstructed track and the position of the Sun $\left(\Psi_{\odot}\right)$ is plotted as function of the reconstructed energy of the event. These PDFs are normalized per solid angle to unity.

$$
\mathrm{TS}=\log _{10}\left(\frac{\mathcal{L}\left(\hat{n}_{\text {sig }}\right)}{\mathcal{L}(0)}\right)
$$


The denominator corresponds to the likelihood of the null hypothesis case, for the backgroundonly scenario. The numerator corresponds to the alternative hypothesis for the background + signal events in the sample, being $\hat{n}_{\text {sig }}$ the best-fit parameter of Eq. 1.

The ANTARES quality cuts are chosen to optimize the sensitivitiy to the SA $v$ flux. Pseudoexperiments are conducted to build TS distributions. $O\left(10^{4}\right)$ pseudo-experiments have been performed for the null and the alternative hypotheses. Each pseudo-experiment consists of mock samples randomly sampled according to the PDF of a certain hypothesis.

In order to introduce the natural statistical fluctuations effect in the pseudo-experiments, each TS distribution is transformed through a Poisson function. Also, a 15\% systematic uncertainty on the number of detected events is expected with ANTARES [16]. This effect is included folding each TS distribution with a Gaussian smearing of $15 \%$ width.

The resulting TS distributions are compared with the median of the background-only distribution to obtain the $90 \%$ confidence level (CL) sensitivity on the number of signal events. This sensitivity represents the minimum number of events $n_{\mathrm{sig}}^{90 \% \mathrm{CL}}$ needed for being able to discriminate from the pure background case, giving a false positive error less or equal than $10 \%$ of the times. According to Neyman, upper limits will be set equal to the sensitivity in case a value smaller than the background median is observed in the data.

In order to convert the number of signal events $n_{\mathrm{sig}}^{90 \% \mathrm{CL}}$ into a sensitivity to a SA $v$ flux, the following expression is used:

$$
\frac{d \Phi_{v_{\mu}}^{90 \% \mathrm{CL}}(E)}{d E}=\frac{n_{\mathrm{sig}}^{90 \% \mathrm{CL}}}{\bar{n}_{\mathrm{sig}}^{\text {theor }}} \frac{d \Phi_{\nu_{\mu}}^{\text {theor }}(E)}{d E}=C_{90} \cdot \frac{d \Phi_{\nu_{\mu}}^{\text {theor }}(E)}{d E},
$$

The first member of the equation stands for the flux upper limit/sensitivity. The second and third members of the equality contains the theoretical flux model multiplied by a scale factor $C_{90}$. This scale factor is defined as the ratio between $n_{\text {sig }}^{90 \%} \mathrm{CL}$, and the expected number of signal events in ANTARES during the data taking period $(T)$ for a given theoretical model. The expected number of signal events $\bar{n}_{\text {sig }}^{\text {theor }}$ is computed integrating the product of the theoretical model flux by the effective area of the detector $\left(A^{\text {eff }}\right)$ over the energy range of interest (Eq. 4):

$$
\bar{n}_{\text {sig }}^{\text {theor }}=T \int \sum_{l \in v_{\nu}, \bar{v}_{\mu}}\left(\frac{d \Phi_{l}^{\text {theor }}\left(E^{\prime}\right)}{d E} A_{l}^{\text {eff }}\left(E^{\prime}\right)\right) d E^{\prime} .
$$

\section{Results}

As mentioned in section 3.1, the base-line case is the combination of the cosmic ray model $H 3 a$ [7] with the solar density profile Ser+Stein [9]. Neutrino oscillations and normal mass ordering, from best fits values, is assumed [1,13]. The Sun is considered as a point source. Only $v_{\mu}$ and $\bar{v}_{\mu}$ arriving at the detector are considered.

Figure 2 shows the event distribution, within the RoI of $30^{\circ}$ from the Sun, for the data (dots) alongside with the expected signal distribution (blue histogram) and background (green histogram) events. The signal distribution has been magnified 100 times for comparative reasons. No excess in data over the expected background is observed. 


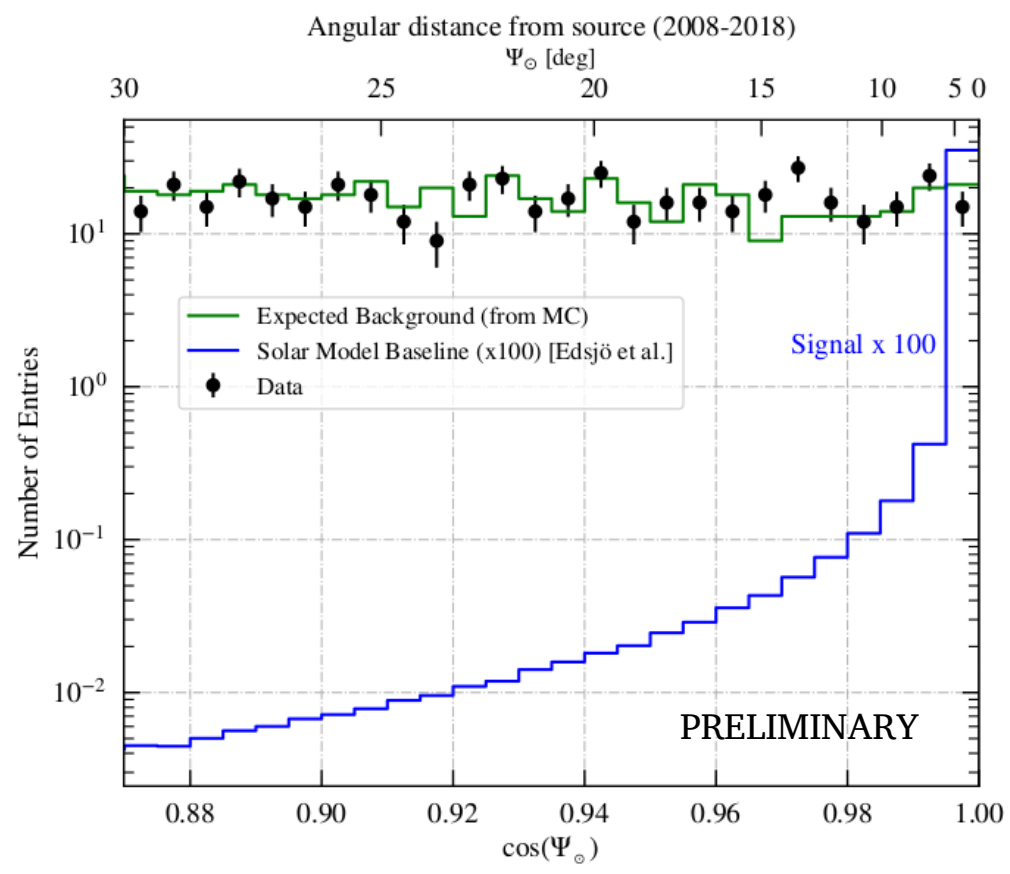

Figure 2: Event distribution as a function of the angular separation $\Psi_{\odot}$ around the source. The expected signal, in blue, is scaled for visibility. The expected background (green histogram) is shown with the data.

The expected number of signal events for this model within the RoI in the 3022 days of lifetime is $\bar{n}_{\text {sig }}^{\text {theor }} \approx 0.366$. The expected number of background events is $\bar{n}_{\text {bkg }}^{\mathrm{MC}}=470$. The number of events in the data sample in the RoI is 461 .

\begin{tabular}{l||lccc} 
Model & Source Shape & $n_{\text {sig, sens }}^{90 \% \mathrm{CL}}$ & $n_{\text {sig, up-lim }}^{90 \% \mathrm{CL}}$ & $p$-val \\
\hline \multirow{3}{*}{ H3a-Ser+Stein [7, 9] } & Point Source & 2.70 & 3.15 & 0.41 \\
& Disk & 2.80 & 3.25 & 0.43 \\
& Ring & 3.45 & 3.45 & 0.5
\end{tabular}

Table 1: Sensitivities and 90\% CL upper-limits for the base-line Solar Atmospheric neutrino model, and for three different Sun shapes considered. The last column is the $p$-values corresponding to the quoted upper limits.

After the data unblinding, the $90 \%$ CL upper limit obtained is $n_{\mathrm{sig}}^{90 \% \mathrm{CL}}=3.15$, and the flux scale factor $C_{90}=8.6$. The scale factor value tells us that in order to exclude the model, we would need a flux 8.6 times larger. The final sensitivity and upper-limit obtained for the H3a-Ser+Stein model is shown in Fig. 3 as a comparison with the theoretical flux model itself and with the Ice Cube latest result [17].

The Gaisser-Stanev-Tilav 4-generation cosmic ray model, refered as GST4, and the Grevesse \& Sauval solar density profile, refered as $\operatorname{Ser}+G S 98$, which are already included within the solar_crnu package, have been tested and the results are within a $2 \%$ difference with respect to the results shown above. 


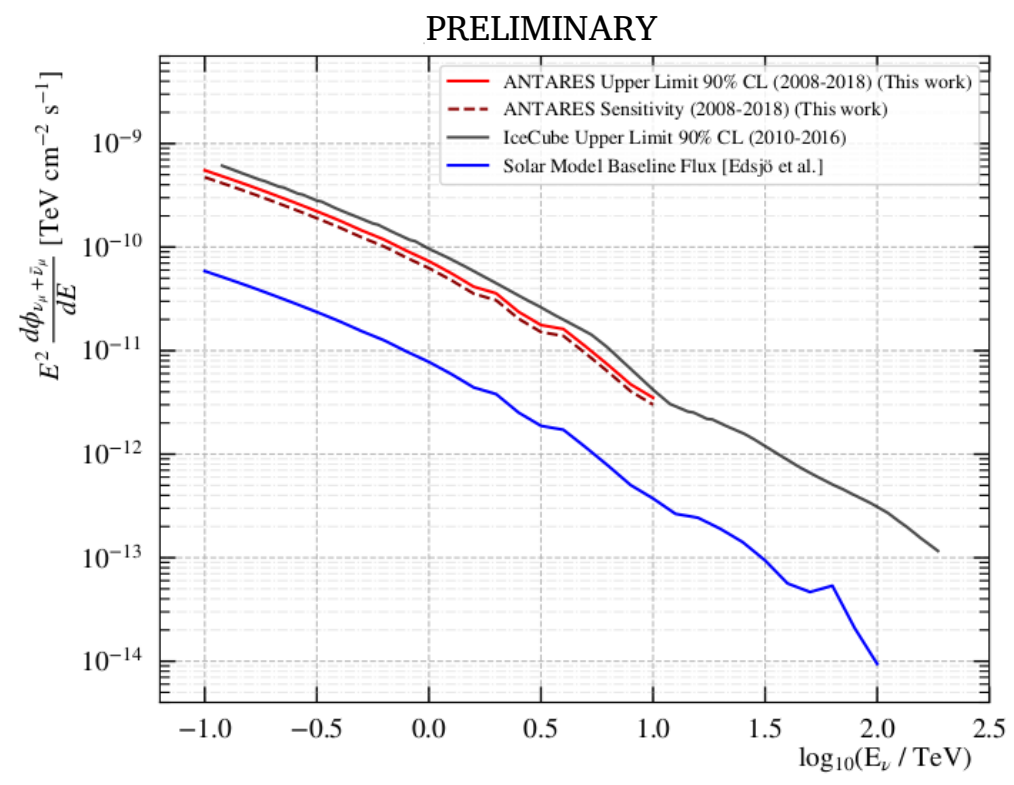

Figure 3: ANTARES upper limit (solid red) for 11 years of data, assuming the Sun as point like source for the base-line model $\mathrm{H} 3 \mathrm{a}$-Ser+Stein (solid blue line). For comparison, the 11 years ANTARES sensitivity (red dashed) and Ice Cube upper limit for 6 years [17] (solid black) are also shown. The ANTARES 90\% expected events falls within the plotted energy range.

\section{Conclusion}

An unbinned likelihood analysis has been performed with 11 years of ANTARES data. The total analysis lifetime is 3022 days. No signal evidence was observed, instead a 90\% CL energy flux upper limit has been established to be about $7 \times 10^{-11}\left[\mathrm{TeV} \mathrm{cm}^{-2} \mathrm{~s}^{-1}\right]$ at $1 \mathrm{TeV}$ neutrino energy with a p-value 0.41 .

\section{Acknowledgements}

We gratefully acknowledge the financial support of Ministerio de Ciencia, Innovación, Investigación y Universidades (MCIU), Programa Estatal de Generación de Conocimiento (ref. PGC2018096663-B-C44,-C41) (MCIU/FEDER) and Junta de Andalucía (ref. A-FQM-053-UGR18), Spain.

\section{References}

[1] J. Edsjö et al., Neutrinos from cosmic ray interactions in the sun, Journal of Cosmology and Astroparticle Physics 2017 (2017) 033.

[2] C. Argüelles et al., Solar atmospheric neutrinos and the sensitivity floor for solar dark matter annihilation searches, Journal of Cosmology and Astroparticle Physics 2017 (2017) 024.

[3] M. Ageron et al., ANTARES: The first undersea neutrino telescope, Nuclear Instruments and Methods in Physics Research Section A: Accelerators, Spectrometers, Detectors and Associated Equipment 656 (2011) 11. 
[4] P. Amram et al., The ANTARES optical module, Nuclear Instruments and Methods in Physics Research Section A: Accelerators, Spectrometers, Detectors and Associated Equipment $\mathbf{4 8 4}$ (2002) 369.

[5] J. Aguilar et al., Performance of the front-end electronics of the ANTARES neutrino telescope, Nuclear Instruments and Methods in Physics Research Section A: Accelerators, Spectrometers, Detectors and Associated Equipment 622 (2010) 59.

[6] A. Albert et al., Monte Carlo simulations for the ANTARES underwater neutrino telescope, Journal of Cosmology and Astroparticle Physics 2021 (2021) 064.

[7] T.K. Gaisser, Spectrum of cosmic-ray nucleons, kaon production, and the atmospheric muon charge ratio, https://arxiv. org/abs/1111.6675.

[8] T.K. Gaisser et al., Cosmic ray energy spectrum from measurements of air showers, Frontiers of Physics 8 (2013) 748.

[9] A.M. Serenelli et al., New solar composition: The problem with solar models revisited, The Astrophysical Journal 705 (2009) L123.

[10] N. Grevesse et al., Standard solar composition, Space Science Reviews 85 (1998) 161.

[11] J. Edsjö et al., WIMPSIM 5.0: WimpAnn, med_dec, solar_crnu and WimpEvent. http://wimpsim.astroparticle.se/code/wimpsim-5.0.pdf.

[12] M. Blennow et al., Neutrinos from WIMP annihilations obtained using a full three-flavor Monte Carlo approach, Journal of Cosmology and Astroparticle Physics 2008 (2008) 021.

[13] I. Esteban et al., Updated fit to three neutrino mixing: exploring the accelerator-reactor complementarity, Journal of High Energy Physics 2017 (2017) .

[14] A. Albert et al., All-flavor search for a diffuse flux of cosmic neutrinos with nine years of ANTARES data, The Astrophysical Journal 853 (2018) L7.

[15] J. Neyman, Outline of a theory of statistical estimation based on the classical theory of probability, Philosophical Transactions of the Royal Society of London. Series A, Mathematical and Physical Sciences 236 (1937) 333.

[16] S. Adrián-Martínez et al., Searh for cosmic neutrino point sources with four years of data from the ANTARES telescope, The Astrophysical Journal 760 (2012) 53.

[17] M. Aartsen et al., Searches for neutrinos from cosmic-ray interactions in the Sun using seven years of IceCube data, Journal of Cosmology and Astroparticle Physics 2021 (2021) 025. 


\section{Full Authors List: ANTARES Collaboration $\neq$}

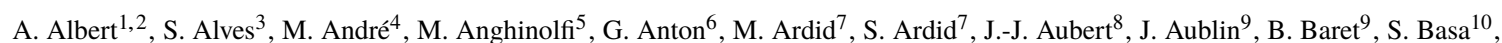

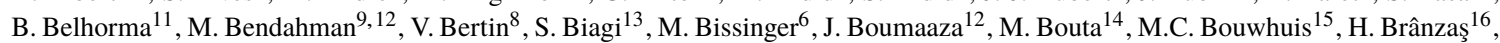
R. Bruijn ${ }^{15,17}$, J. Brunner ${ }^{8}$, J. Busto ${ }^{8}$, B. Caiff ${ }^{5}$, A. Capone ${ }^{18,19}$, L. Caramete ${ }^{16}$, J. Carr ${ }^{8}$, V. Carretero ${ }^{3}$, S. Celli ${ }^{18,19}$, M. Chabab ${ }^{20}$, T. N. Chau ${ }^{9}$, R. Cherkaoui El Moursli ${ }^{12}$, T. Chiarusi ${ }^{21}$, M. Circella $^{22}$, A. Coleiro ${ }^{9}$, M. Colomer-Molla ${ }^{9,3}$, R. Coniglione $^{13}$, P. Coyle $^{8}$, A. Creusot $^{9}$, A. F. Díaz ${ }^{23}$, G. de Wasseige ${ }^{9}$, A. Deschamps ${ }^{24}$, C. Distefano ${ }^{13}$, I. Di Palma ${ }^{18,19}$, A. Domi ${ }^{15,17}$, C. Donzaud ${ }^{9,25}$, D. Dornic ${ }^{8}$, D. Drouhin ${ }^{1,2}$, T. Eberl ${ }^{6}$, T. van Eeden ${ }^{15}$, D. van Eijk ${ }^{15}$, N. El Khayati ${ }^{12}$, A. Enzenhöfer ${ }^{8}$, P. Fermani ${ }^{18,19}$, G. Ferrara $^{13}$, F. Filippini ${ }^{21,26}$, L.A. Fusco ${ }^{8}$, Y. Gatelet ${ }^{9}$, P. Gay ${ }^{27,9}$, H. Glotin $^{28}$, R. Gozzini ${ }^{3}$, R. Gracia Ruiz ${ }^{15}$, K. Graf ${ }^{6}$, C. Guidi ${ }^{5,29}$, S. Hallmann ${ }^{6}$, H. van Haren ${ }^{30}$, A.J. Heijboer ${ }^{15}$, Y. Hello ${ }^{24}$, J.J. Hernández-Rey ${ }^{3}$, J. Höß1 ${ }^{6}$, J. Hofestädt ${ }^{6}$, F. Huang ${ }^{8}$, G. Illuminati ${ }^{9}, 21,26$, C.W James $^{31}$, B. Jisse-Jung ${ }^{15}$, M. de Jong ${ }^{15,32}$, P. de Jong ${ }^{15}$, M. Kadler ${ }^{33}$, O. Kalekin ${ }^{6}$, U. Katz ${ }^{6}$, N.R. Khan-Chowdhury ${ }^{3}$, A. Kouchner ${ }^{9}$, I. Kreykenbohm ${ }^{34}$, V. Kulikovskiy ${ }^{5,36}$, R. Lahmann ${ }^{6}$, R. Le Breton ${ }^{9}$, D. Lefèvre ${ }^{35}$, E. Leonora ${ }^{36}$, G. Levi ${ }^{21,26}$, M. Lincetto ${ }^{8}$, D. Lopez-Coto ${ }^{37}$, S. Loucatos ${ }^{38,9}$, L. Maderer ${ }^{9}$, J. Manczak ${ }^{3}$, M. Marcelin ${ }^{10}$, A. Margiotta ${ }^{21,26}$, A. Marinelli ${ }^{39}$, J.A. Martínez-Mora ${ }^{7}$, K. Melis ${ }^{15,17}$, P. Migliozzi ${ }^{39}$, A. Moussa ${ }^{14}$, R. Muller ${ }^{15}$, L. Nauta ${ }^{15}$, S. Navas ${ }^{37}$, E. Nezri ${ }^{10}$, B. O' Fearraigh $^{15}$, A. Păun ${ }^{16}$, G.E. Păvălaş ${ }^{16}$, C. Pellegrino ${ }^{21,40,41}$, M. Perrin-Terrin ${ }^{8}$, V. Pestel ${ }^{15}$, P. Piattelli ${ }^{13}$, C. Pieterse ${ }^{3}$, C. Poirè ${ }^{7}$, V. Popa ${ }^{16}$, T. Pradier ${ }^{1}$, N. Randazzo ${ }^{36}$, S. Reck ${ }^{6}$, G. Riccobene ${ }^{13}$, A. Romanov ${ }^{5,29}$, A. Sánchez-Losa ${ }^{3,22}$, F. Salesa Greus ${ }^{3}$, D. F. E. Samtleben ${ }^{15,32}$, M. Sanguineti ${ }^{5,29}$, P. Sapienza ${ }^{13}$, J. Schnabel ${ }^{6}$, J. Schumann ${ }^{6}$, F. Schüssler ${ }^{38}$, M. Spurio ${ }^{21,26}$, Th. Stolarczyk ${ }^{38}$, M. Taiuti ${ }^{5,29}$, Y. Tayalati ${ }^{12}$, S.J. Tingay ${ }^{31}$, B. Vallage ${ }^{38,9}$, V. Van Elewyck ${ }^{9,41}$, F. Versari ${ }^{21,26,9}$, S. Viola ${ }^{13}$, D. Vivolo ${ }^{39,43}$, J. Wilms ${ }^{34}$, S. Zavatarelli ${ }^{5}$, A. Zegarelli ${ }^{18,19}$, J.D. Zornoza ${ }^{3}$, and J. Zúñiga ${ }^{3}$

${ }^{1}$ Université de Strasbourg, CNRS, IPHC UMR 7178, F-67000 Strasbourg, France. ${ }^{2}$ Université de Haute Alsace, F-68100 Mulhouse, France. ${ }^{3}$ IFIC - Instituto de Física Corpuscular (CSIC - Universitat de València) c/ Catedrático José Beltrán, 2 E-46980 Paterna, Valencia, Spain. ${ }^{4}$ Technical University of Catalonia, Laboratory of Applied Bioacoustics, Rambla Exposició, 08800 Vilanova i la Geltrú, Barcelona, Spain. ${ }^{5}$ INFN - Sezione di Genova, Via Dodecaneso 33, 16146 Genova, Italy. ${ }^{6}$ Friedrich-Alexander-Universität Erlangen-Nürnberg, Erlangen Centre for Astroparticle Physics, Erwin-Rommel-Str. 1, 91058 Erlangen, Germany. ${ }^{7}$ Institut d'Investigació per a la Gestió Integrada de les Zones Costaneres (IGIC) - Universitat Politècnica de València. C/ Paranimf 1, 46730 Gandia, Spain. ${ }^{8}$ Aix Marseille Univ, CNRS/IN2P3, CPPM, Marseille, France. ${ }^{9}$ Université de Paris, CNRS, Astroparticule et Cosmologie, F-75013 Paris, France. ${ }^{10}$ Aix Marseille Univ, CNRS, CNES, LAM, Marseille, France. ${ }^{11}$ National Center for Energy Sciences and Nuclear Techniques, B.P.1382, R. P.10001 Rabat, Morocco. ${ }^{12}$ University Mohammed V in Rabat, Faculty of Sciences, 4 av. Ibn Battouta, B.P. 1014, R.P. 10000 Rabat, Morocco. ${ }^{13}$ INFN - Laboratori Nazionali del Sud (LNS), Via S. Sofia 62, 95123 Catania, Italy. ${ }^{14}$ University Mohammed I, Laboratory of Physics of Matter and Radiations, B.P.717, Oujda 6000, Morocco. ${ }^{15}$ Nikhef, Science Park, Amsterdam, The Netherlands. ${ }^{16}$ Institute of Space Science, RO-077125 Bucharest, Măgurele, Romania. ${ }^{17}$ Universiteit van Amsterdam, Instituut voor Hoge-Energie Fysica, Science Park 105, 1098 XG Amsterdam, The Netherlands. ${ }^{18}$ INFN - Sezione di Roma, P.le Aldo Moro 2, 00185 Roma, Italy. ${ }^{19}$ Dipartimento di Fisica dell’Università La Sapienza, P.le Aldo Moro 2, 00185 Roma, Italy. ${ }^{20}$ LPHEA, Faculty of Science - Semlali, Cadi Ayyad University, P.O.B. 2390, Marrakech, Morocco. ${ }^{21}$ INFN - Sezione di Bologna, Viale Berti-Pichat 6/2, 40127 Bologna, Italy. ${ }^{22}$ INFN - Sezione di Bari, Via E. Orabona 4, 70126 Bari, Italy. ${ }^{23}$ Department of Computer Architecture and Technology/CITIC, University of Granada, 18071 Granada, Spain. ${ }^{24}$ Géoazur, UCA, CNRS, IRD, Observatoire de la Côte d'Azur, Sophia Antipolis, France. ${ }^{25}$ Université Paris-Sud, 91405 Orsay Cedex, France. ${ }^{26}$ Dipartimento di Fisica e Astronomia dell'Università, Viale Berti Pichat 6/2, 40127 Bologna, Italy. ${ }^{27}$ Laboratoire de Physique Corpusculaire, Clermont Université, Université Blaise Pascal, CNRS/IN2P3, BP 10448, F-63000 Clermont-Ferrand, France. ${ }^{28}$ LIS, UMR Université de Toulon, Aix Marseille Université, CNRS, 83041 Toulon, France. ${ }^{29}$ Dipartimento di Fisica dell'Università, Via Dodecaneso 33, 16146 Genova, Italy. ${ }^{30}$ Royal Netherlands Institute for Sea Research (NIOZ), Landsdiep 4, $1797 \mathrm{SZ}$ 't Horntje (Texel), the Netherlands. ${ }^{31}$ International Centre for Radio Astronomy Research - Curtin University, Bentley, WA 6102, Australia. ${ }^{32}$ Huygens-Kamerlingh Onnes Laboratorium, Universiteit Leiden, The Netherlands. ${ }^{33}$ Institut für Theoretische Physik und Astrophysik, Universität Würzburg, Emil-Fischer Str. 31, 97074 Würzburg, Germany. ${ }^{34}$ Dr. Remeis-Sternwarte and ECAP, Friedrich-Alexander-Universität Erlangen-Nürnberg, Sternwartstr. 7, 96049 Bamberg, Germany. ${ }^{35}$ Mediterranean Institute of Oceanography (MIO), Aix-Marseille University, 13288, Marseille, Cedex 9, France; Université du Sud Toulon-Var, CNRS-INSU/IRD UM 110, 83957, La Garde Cedex, France. ${ }^{36}$ INFN - Sezione di Catania, Via S. Sofia 64, 95123 Catania, Italy. ${ }^{37}$ Dpto. de Física Teórica y del Cosmos \& C.A.F.P.E., University of Granada, 18071 Granada, Spain. ${ }^{38}$ IRFU, CEA, Université Paris-Saclay, F-91191 Gif-sur-Yvette, France. ${ }^{39}$ INFN - Sezione di Napoli, Via Cintia 80126 Napoli, Italy. ${ }^{40}$ Museo Storico della Fisica e Centro Studi e Ricerche Enrico Fermi, Piazza del Viminale 1, 00184, Roma. ${ }^{41}$ INFN - CNAF, Viale C. Berti Pichat 6/2, 40127, Bologna. ${ }^{42}$ Institut Universitaire de France, 75005 Paris, France. ${ }^{43}$ Dipartimento di Fisica dell'Università Federico II di Napoli, Via Cintia 80126, Napoli, Italy. 